\title{
Initial steps to stabilize the microenvironment for implantation of stem/progenitor cells in diseased renal parenchyma
}

Will W. Minuth* and Lucia Denk

*Correspondence: will.minuth@vkl.uni-regensburg.de

Molecular and Cellular Anatomy, University of Regensburg, Regensburg, Germany.

\begin{abstract}
Stem/progenitor cells are in the focus of research as a therapeutic perspective to cure acute and chronic renal failure. Following this innovative strategy one has to consider that stem/progenitor cells are first kept in the beneficial atmosphere of a $\mathrm{CO}_{2}$-dependent culture medium, while after implantation they are exposed to unbalanced interstitial fluid of diseased parenchyma. However, this abrupt transition has consequences, since it limits survival of implanted stem/progenitor cells. To offer a constant environment during isolation, culture, surgical processing and initial seeding within diseased parenchyma, an improved concept is under current work. In present experiments stabilization of fluid environment was investigated by isolating renal stem/progenitor cells in chemically defined and $\mathrm{CO}_{2}$-stabilized culture media. Interstitial influences on spatial development of tubules were tested by mounting stem/progenitor cells in a polyester fleece as a substitute for extracellular matrix and by transporting always fresh medium in perfusion culture for 13 days. Finally, morphological quality of raised constructs was analyzed by histochemistry, light and transmission electron microscopy. The present results demonstrate that three different culture media can be short-listed for protecting stem/progenitor cells during the process of implantation. Earlier approved Iscove's Modified Dulbecco's Medium buffered by HEPES revealed formation of numerous tubules. Also application of Leibovitz's L-15 Medium and $\mathrm{CO}_{2}$ Independent Medium demonstrated unexpected promoting effects on the generation of tubules. In all series typical features of transporting tubule cells were registered. Formation of an excess of vacuoles as an indicator for cytotoxicity was not observed. In conclusion, although very different in chemical composition the tested culture media reflect an advantageous microenvironment for isolation, implantation and development of stem/ progenitor cells.
\end{abstract}

Keywords: Kidney, stem/progenitor cells, implantation, artificial interstitium, perfusion culture, chemically defined culture medium

\section{Introduction}

A series of papers published in the course of the last years demonstrates that a stem/progenitor cell-based therapy appears as an attractive option to cure acute and chronic renal failure [1-4]. However, critical reading of related literature also elucidates that this innovative therapeutic approach is still in a phase of basic research, since survival of implanted stem/ progenitor cells and initial regeneration is limited [5-7]. This fact has several causes.

Implantation of stem/progenitor cells can be principally performed by infusion via the blood vessel system $[8,9]$ or by punctual implantation into the diseased parenchyma of the kidney $[10,11]$. An alternative approach is to implant them in the space left between the organ capsule and the outer parenchyma at the earlier site of nephron formation [12]. The different surgical strategies generally reflect that there are difficulties to transfer a successful amount of stem/progenitor cells to the site of repair and that an effective implantation technique is up to date not found.

A further problem is the seeding of stem/progenitor cells during implantation and the phase of initial regeneration within the environment of diseased parenchyma. One has to consider that development of intact parenchyma needs not only potent stem/progenitor cells but also stimulating interstitial fluid and attractive extracellular matrix (Figure 1a). When these parameters complement each other, intact development can be expected (Figure $\mathbf{1 b}$ ). However, after implantation into diseased renal parenchyma stem/progenitor cells are exposed to degenerating nephrons, altered extracellular matrix and to unbalanced electrolytes, growth factors, interleukins or hormones occurring in interstitial fluid $[13,14]$. Further a series of metabolites is causing inflammation so that repair is prevented [15-18]. Finally, the interstitial fluid within diseased parenchyma shows lack of oxygen and accumulation of $\mathrm{CO}_{2}$ due to damage of peritubular capillaries [19]. It is obvious that such an environment is not featuring regeneration (Figure 1c).

In the described harmful atmosphere implanted stem/ progenitor cells have to survive and must turn the situation so that repair of parenchyma becomes possible $[20,21]$. In consequence, to prevent damage of stem/progenitor cells, a technical solution might be to mount them before implantation in a polyester fleece pad $[\mathbf{2 2 , 2 3 ]}$. In this scenario the fleece fibers mimic extracellular matrix for implanted stem/progenitor cells, while the space between acts as a reservoir for culture medium and in turn as a fence against surrounding interstitial fluid of diseased parenchyma. At least for the initial period of 


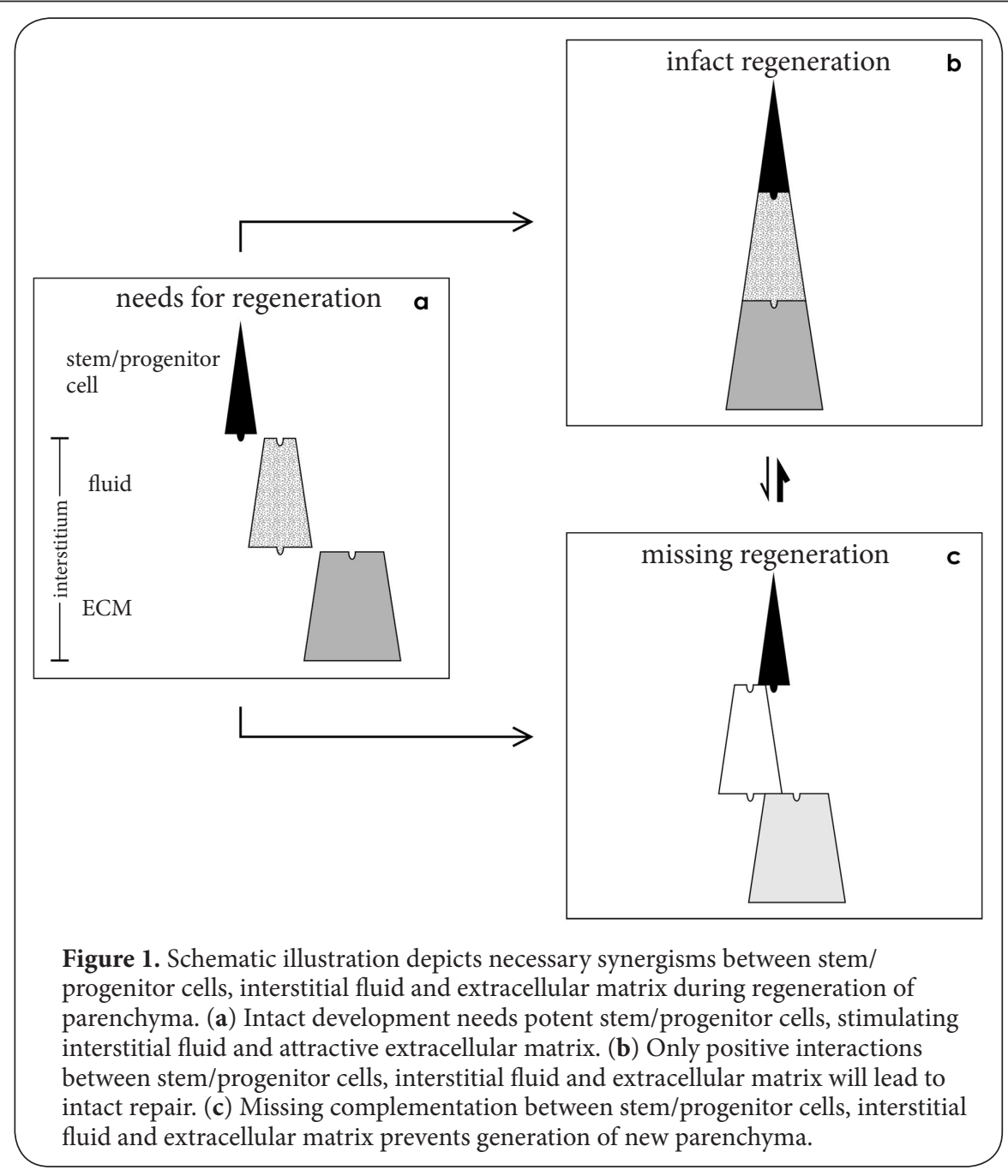

seeding the medium within the fleece pad is available so that stem/progenitor cells are provided with nutrition and respiratory gas. Applying this technique the volume must be big enough to support initial development of implanted stem/progenitor cells and to compensate instability of $\mathrm{pH}$ or influences of unbalanced metabolites derived from surrounding parenchyma.

It is surprising that only little information is available about reactions of stem/progenitor cells coming in contact with altered fluid environment [24]. In consequence, to obtain more insights the actual experiments were performed. Renal stem/ progenitor cells were mounted in a polyester interstitium and exposed during perfusion culture to three media containing $\mathrm{CO}_{2}$-stabilized buffer systems. Quality of regenerated tubules was then recorded by cell biological methods.

\section{Methods}

\section{Isolation of renal stem/progenitor cells}

Both kidneys from one-day old anesthesized and sacrificed New Zealand rabbits (Seidl, Oberndorf, Germany) were removed under sterile conditions and cut into ventral and dorsal halfs as earlier described $[22,23]$. By stripping off the capsula fibrosa with fine forceps a constantly thin layer of stem/progenitor cell niches is adherent to the explant. By this method an embryonic tissue layer of up to $1 \mathrm{~cm}$ in square can be harvested.

\section{Creation of an artificial interstitium}

To investigate spatial development of tubules an isolated embryonic tissue layer containing numerous renal stem/ progenitor niches was mounted in an artificial interstitium as earlier described (Figure 2a) [25]. Briefly, the isolated tissue was placed between punched out pieces of polyester fleece (17, Walraf, Grevenbroich, Germany) and fixed inside a Minusheet ${ }^{\circledR}$ tissue carrier with $13 \mathrm{~mm}$ inner diameter (Minucells and Minutissue, Bad Abbach, Germany). Then the tissue carrier was transferred to a perfusion culture container with horizontal flow characteristics (Figure $\mathbf{2 b}$ ). For a period of 13 days always fresh medium was continuously transported at a rate of 1.25 $\mathrm{ml} / \mathrm{h}$ with an IPC N8 peristaltic pump (Ismatec, Wertheim, 
a

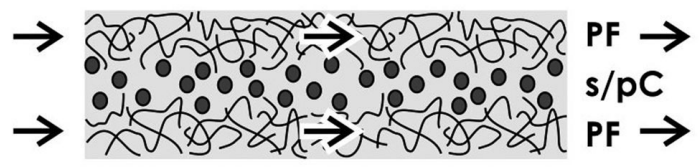

b

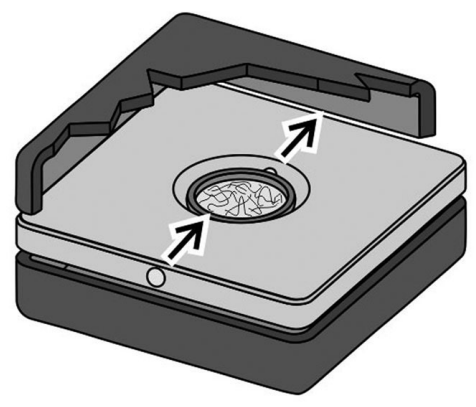

c

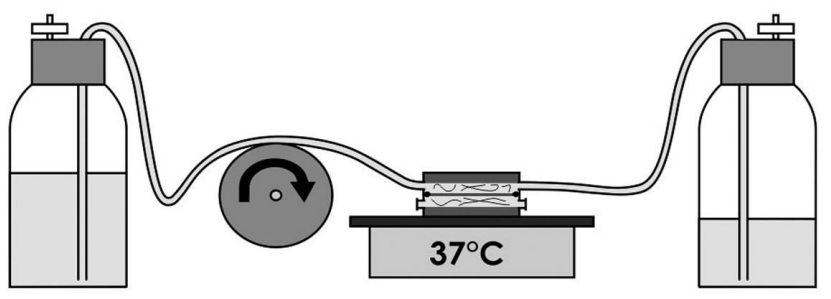

Figure 2. Schematic illustration shows generation of tubules with renal stem/progenitor cells and an artificial interstium in perfusion culture. (a) Isolated renal stem/ progenitor cells $(\mathrm{s} / \mathrm{pC})$ are mounted between layers of polyester fleece to create an artificial interstitium. In the space between the polyester fibers (PF) exchange of culture medium (arrow) takes place. (b) The fleece layers are fixed in a Minusheet ${ }^{\circledR}$ tissue carrier, which is placed in a perfusion culture container with horizontal flow conditions. (c) Perfusion culture is performed for 13 days under atmospheric air. Always fresh culture medium is transported by a peristaltic pump $(1.25 \mathrm{ml} / \mathrm{h})$ from a storage bottle to the container and then to a waste bottle.

Germany) (Figure 2c). All of the culture experiments were performed under atmospheric air.

\section{Applied chemically defined culture media}

To generate tubules chemically defined Iscove's Modified Dulbecco's Medium (IMDM, Nr. 21980-032, 14 cultured specimens), Leibovitz's L-15 Medium (Nr. 31415-029, 15 cultured specimens) and $\mathrm{CO}_{2}$ Independent Medium (Nr. 18045054, 19 cultured specimens) all including Phenolred (GIBCO/ Invitrogen, Karlsruhe, Germany) were applied. Infections were prevented by adding an antibiotic-antimycotic cocktail ( $1 \%$, GIBCO/Invitrogen). Tubulogenic development was induced by application of aldosterone $\left(1 \times 10^{-7} \mathrm{M}\right.$, Fluka, Taufkirchen, Germany) as earlier described [22]. To reach a constant $\mathrm{pH}$ of 7.4 under atmospheric air $\mathrm{N}-2$-Hydroxyethylpiperazine-N-2-
Ethane Sulfonic Acid (HEPES; GIBCO/Invitrogen) was added by titration in necessary amounts.

\section{Histochemistry on generated tubules}

After run of a perfusion culture experiment the polyester fleece with adherent renal tubules was fixed in $70 \%$ ethanol, then whole mount label with fluorescent Soybean Agglutinin (SBA, Vector, Burlingame, USA) was performed as earlier described [22]. On cryosections BPL (Bauhinia Purpurea Lectin), GSL (Griffonia Simplicifolia Lectin), LTL (Lotus Tetragonolobus Lectin), WGA (Wheat Germ Agglutinin), DBA (Dolichos Biflorus Agglutinin), PNA (Peanut Agglutinin) and SBA was tested. All lectins were obtained from Vector.

\section{Transmission electron microscopy}

To investigate semithin and ultrathin sections of generated tubules, specimens were transferred to immersion fixation in $5 \%$ glutaraldehyde (Serva, Heidelberg, Germany) buffered with $0.15 \mathrm{M}$ sodium cacodylate at a $\mathrm{pH}$ of 7.4. Fixation was made for 1 day at room temperature. After several washes with $0.15 \mathrm{M}$ sodium cacodylate the specimens were postfixed in the same buffer but containing additionally $1 \%$ osmium tetroxide (Science Services, München, Germany). In a next step the tissue was rinsed with sodium cacodylate buffer and dehydrated in graded series of ethanol. At the end specimens were embedded in Epon (Fluka, Taufkirchen, Germany) polymerizing at $60^{\circ} \mathrm{C}$ for $48 \mathrm{~h}$.

Semithin and ultrathin sections were made with a diamond knife on an ultramicrotome Ultracut E (Reichert-Jung, Nussloch, Germany). The semithin sections were stained with Richardson solution and then analyzed by using a Leica DM 750 microscope (Leica, Wetzlar, Germany). Images were taken with a digital camera from Leica (ICC50 HD) and thereafter processed with Corel DRAW Graphic Suite X5 (Corel Corporation, Otawa, Canada).

The obtained ultrathin sections were collected onto slot grids (Plano, Wetzlar, Germany) coated with $1.5 \%$ pioloform (Plano) and contrasted using $2 \%$ uranyl acetate and lead citrate (Fluka) as earlier described [26]. The samples were then examined at $80 \mathrm{kV}$ using an EM 902 transmission electron microscope (Zeiss, Oberkochen, Germany).

\section{Definition of cells within the renal stem/progenitor cell niche}

For the presented experiments embryonic tissue derived from the outer cortex of neonatal rabbit kidney was analyzed containing renal stem/progenitor cell niches. The nomenclature of previously published papers was used $[23,27]$.

\section{Related ethical rules}

Performed experiments are in accordance with the Animal Ethics Committee, University of Regensburg, Regensburg, Germany. Regarding a future implantation of described polyester fleece pads in human kidney any coating with 

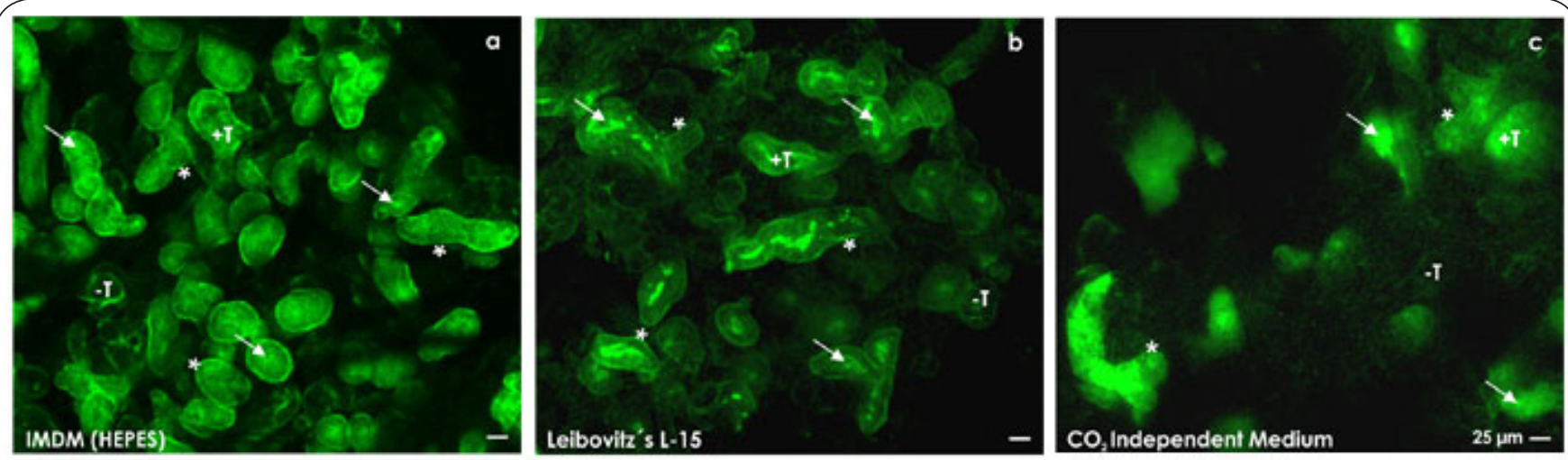

Figure 3. Fluorescence microscopy on whole mount species labeled with SBA. Generated tubules (T) were kept for 13 days in perfusion culture. Most intensive label is detected after culture in (a) Iscove's Modified Dulbecco's Medium (IMDM), while fewer label is found after administration of (b) Leibovitz's L-15 Medium and (c) $\mathrm{CO}_{2}$ Independent Medium. Intact tubules are recognized by a lumen (arrow), an intact epithelium and a covering basal lamina (asterisk). $+\mathrm{T} /$ tubules positive for SBA, $-\mathrm{T} /$ tubules negative for SBA.

biohazardous extracellular matrix molecules was omitted. To perform culture experiments under controlled conditions exclusively chemically defined media without any serum supplementation were applied.

\section{Amount of analyzed specimens}

For the present investigation a total of 48 generated specimens and in each series at least 3 individual samples were morphologically analyzed. In all micrographs the typical biological mean is represented.

\section{Results}

The present set of experiments was performed to investigate development of renal stem/progenitor cells kept under atmospheric air from the point of isolation up to the harvest in $\mathrm{CO}_{2}$-stabilized culture media. To support spatial generation of tubules fibers of a polyester fleece served as substitute for interstitial extracellular matrix during run of perfusion culture over 13 days. The effects of different interstitial fluids on mounted stem/progenitor cells was investigated by Leibovitz's $\mathrm{L}-15$ Medium and $\mathrm{CO}_{2}$ Independent Medium in comparison to earlier used Iscove's Modified Dulbecco's Medium [22-24].

\section{Fluorescence microscopy on generated tubules}

After 13 days of perfusion culture whole mount label was performed to analyze spatial development of regenerated tubules (Figure 3). Fluorescence microscopy shows after Soybean Agglutinin (SBA) label that numerous collecting duct tubules are developing between the polyester fibers of the artificial interstitium as it was described earlier $[\mathbf{2 2 , 2 3 ]}$. It can be recognized that they exhibit a lumen and are covered by a continuously developed basal lamina. By the first view the results demonstrate that tubules can principally develop in the presence of all three applied culture media. However, the relation between intensively labeled and non-labeled tubules differs. Bright SBA label was detected in specimens generated in Iscove's Modified Dulbecco's Medium (Figure 3a), while less labeled tubules were registered after culture in Leibovitz's L-15 Medium (Figure 3b). Only relatively few labeled ones were detected in specimens generated in $\mathrm{CO}_{2}$ Independent Medium (Figure 3c). Further up to date it cannot be explained why samples kept in $\mathrm{CO}_{2}$ Independent Medium illustrate a more diffuse than sharp labeling profile of epithelial cells.

In addition to collecting duct tubules other nephron segments may be contained in generated specimens. Consequently, the binding profile of markers was tested recognizing proximal tubule cells (Table 1). Astonishingly, only a barely visible reaction on single tubules was found in series labeled by BPL, while no label was detected in series with GSL, LTL and WGA.

In contrast, application of DBA showed a faint reaction on specimens generated in Iscove's Modified Dulbecco's Medium, while a barely visible reaction was detected in samples with Leibovitz's L-15 Medium. A faint respectively barely visible label was seen after PNA incubation in specimens cultured with Iscove's Modified Dulbecco's Medium and $\mathrm{CO}_{2}$ Independent Medium. These results clearly demonstrate that no proximal but distal nephron segments are developing.

\section{Light microscopic analysis}

In the next set of experiments semithin sections were cut to obtain more detailed information about morphological features of generated tubules. Specimens generated as well in Iscove's Modified Dulbecco's Medium (Figures 3a and 4a) as in Leibovitz's L-15 (Figures $\mathbf{3 b}$ and $\mathbf{5 a}$ ) and $\mathrm{CO}_{2}$ Independent Medium (Figures $\mathbf{3 c}$ and $\mathbf{5 d}$ ) illustrate development of numerous tubules exhibiting a basal lamina and a lumen. Differences in morphological quality of tubule cells cannot be recognized by light microscopy. Regarding the heterogeneous composition 
Minuth et al. Transplantation Technology 2013,

Table 1. Fluorescence microscopy on generated tubules labeled by lectins to recognize site-specific nephron segments. Label indicates $(+++)$ intense reaction on numerous tubules, $(++)$ faint reaction on single tubules, $(+)$ barely visible reaction on single tubules respectively single cells or (-) negative reaction.

\begin{tabular}{|c|c|c|c|c|}
\hline Target & Marker & $\begin{array}{c}\text { Iscove's Modified } \\
\text { Dulbecco's Medium }\end{array}$ & $\begin{array}{c}\text { Leibovitz's L-15 } \\
\text { Medium }\end{array}$ & $\begin{array}{c}\mathrm{CO}_{2} \text { Independent } \\
\text { Medium }\end{array}$ \\
\hline Proximal tubule & $\begin{array}{l}\text { BPL } \\
\text { Bauhinia Purpurea Lectin }\end{array}$ & + & + & + \\
\hline Proximal tubule & $\begin{array}{l}\text { GSL } \\
\text { Griffonia Simplicifolia Lectin }\end{array}$ & - & - & - \\
\hline Proximal tubule & $\begin{array}{l}\text { LTL } \\
\text { Lotus Tetragonolobus Lectin }\end{array}$ & - & - & - \\
\hline Proximal/Collecting tubule & $\begin{array}{l}\text { WGA } \\
\text { Wheat Germ Agglutinin }\end{array}$ & - & - & - \\
\hline Collecting tubule & $\begin{array}{l}\text { DBA } \\
\text { Dolichos Biflorus Agglutinin }\end{array}$ & ++ & + & - \\
\hline Collecting tubule & $\begin{array}{l}\text { PNA } \\
\text { Peanut Agglutinin }\end{array}$ & ++ & - & + \\
\hline Collecting tubule & $\begin{array}{l}\text { SBA } \\
\text { Soybean Agglutinin }\end{array}$ & +++ & ++ & ++ \\
\hline
\end{tabular}

of offered culture media it speaks in favor that renal stem/ progenitor cells obviously can tolerate a wide spectrum of interstitial fluid. In addition, vacuoles in the cytoplasm of generated tubule cells are rare. Consequently, the results indicate that toxic effects or non appropriate chemical compounds are not present in these selected culture media so that they do not harm stem/progenitor cells during development of tubules.

\section{Electron microscopy}

Transmission electron microscopy (TEM) elucidates that perfusion culture in combination with Iscove's Modified Dulbecco's Medium (Figures 4b and 4c), Leibovitz's L-15 (Figures $\mathbf{5 b}$ and $\mathbf{5 c}$ ) and $\mathrm{CO}_{2}$ Independent Medium (Figures 5e and $\mathbf{5 f}$ ) produces tubules exhibiting intact morphology including an inconspicuously looking cytoplasm. The performed experiments illustrate that application of all three media results in development of tubules containing a polarized epithelium. In each of the cases it was seen that a luminal side faces a clearly visible lumen, while the basal side rests on a continuously developed basal lamina consisting of a lamina rara, lamina densa and lamina fibroreticularis. Furtheron, between the luminal and lateral plasma membranes an intact tight junctional complex is developed consisting of a zonula occludens, zonula adhaerens and desmosome (Figures $\mathbf{4} \mathbf{b}^{\prime}, \mathbf{5} \mathbf{b}^{\prime}$ and $\left.\mathbf{5} \mathbf{e}^{\prime}\right)$. This result points out that a physiological sealing between neighboring cells is established.

Most interestingly are the lateral plasma membranes of tubules generated in Iscove's Modified Dulbecco's Medium (Figure 4c), Leibovitz's L-15 (Figure 5c) and $\mathrm{CO}_{2}$ Independent Medium (Figure 5f). The intercellular space is not pathologically extended but appears to be narrow. In all of the cases a close basal slit is found at the contact site between the basal and lateral plasma membranes. Further at the lateral plasma membranes interdigitating foldings respectively protrusions are found. Most conspicuous is the increased amount of protrusions in species generated by Iscove's Modified Dulbecco's Medium (Figure 4c; 18 in average) and Leibovitz's L-15 Medium (Figure 5c; 15 in average), but they are detected to a lower degree after administration of $\mathrm{CO}_{2}$ Independent Medium (Figure 5f; 11 in average). In so far the occurrence of interdigitating protrusions at the lateral plasma membranes is an important hint that a hypertrophy is developed and that intense physiological transport occurs at this site.

\section{Discussion}

Actual biomedical research illustrates that a discrepancy exists between the hope to cure severe renal failure by implantation of stem/progenitor cells and the reality, which exhibits a limited survival of implanted stem/progenitor cells including incomplete repair of parenchyma [7]. However, due to little experimental experiences it cannot be decided yet, what are the major and what are the minor detrimental influences limiting the process of regeneration.

\section{Maintenance of environment}

Regarding stem/progenitor cell development one of the critical issues is the exposure to successive alterations of microenvironment during handling and implantation. Generally the process of implantation is started with stem/ progenitor cells kept in the atmosphere of a $\mathrm{CO}_{2}$-incubator (Figure 6a). However, the beneficial environment of a $\mathrm{CO}_{2}-$ dependent culture medium is changing, when they are taken out to be rinsed and concentrated for infusion respectively injection. Usually this work is performed in the laboratory under atmospheric air. Due to the low content of $0.03 \%$ $\mathrm{CO}_{2}$ in atmospheric air versus $5 \%$ in a $\mathrm{CO}_{2}$-incubator the contained cells are damaged within minutes by $\mathrm{pH}$ shifting from a neutral into an alkaline range. When an implantation has been performed, the environment is changing again. 

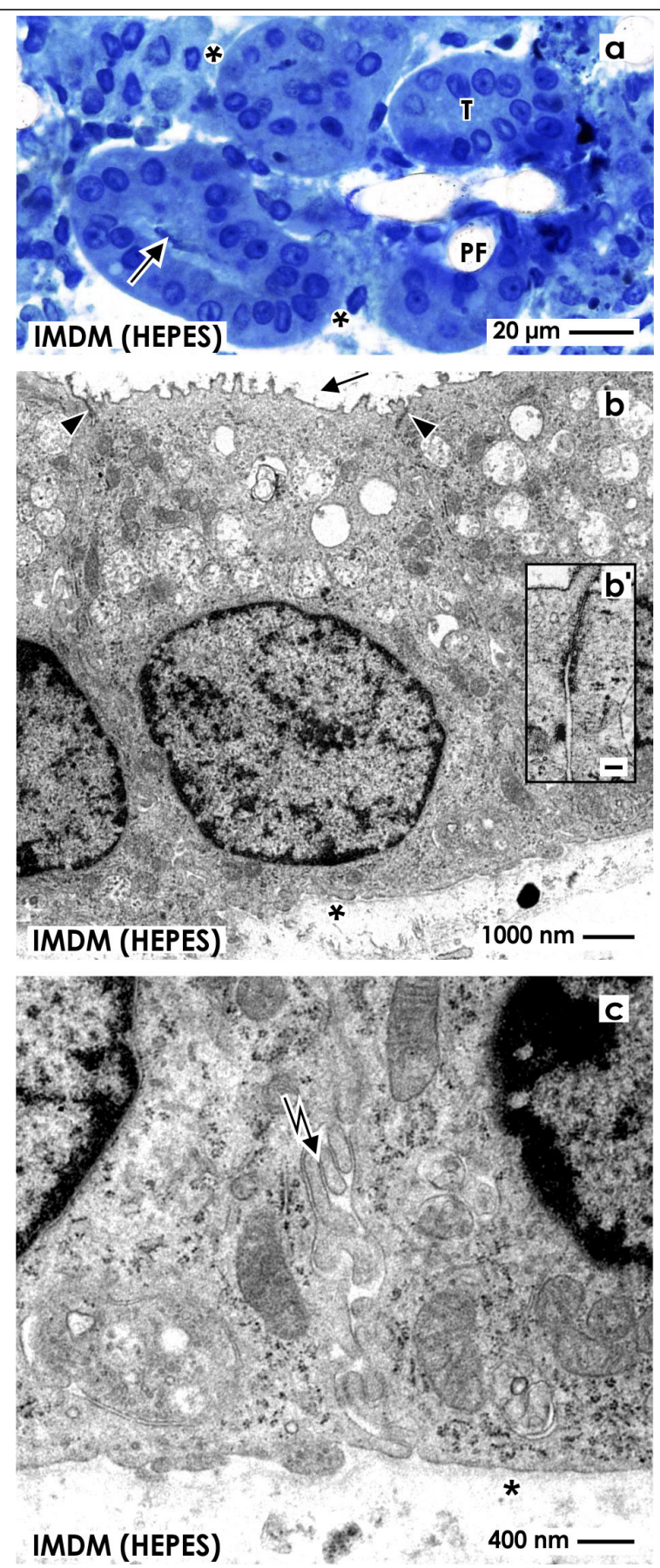

Figure 4. Light and electron microscopy of renal tubules (T) generated in Iscove's Modified Dulbecco's Medium (IMDM) after 13 days. (a) A semithin section depicts between polyester fibers (PF) tubules exhibiting a lumen and a basal lamina. (b) Electron microscopy shows a polarized epithelium within generated tubules. The luminal and lateral plasma membranes are separated by a tight junctional complex (arrow head and b'; bar 100 $\mathrm{nm}$ ). (c) Higher magnification in electron microscopy demonstrates at the lateral plasma membranes intense protrusions (serrated arrow) interdigitating with each other. Lumen is indicated by an arrow, while the basal lamina is labeled by an asterisk.
Now stem/progenitor cells are exposed to harmful interstitial fluid, altered extracellular matrix and a low content of oxygen within diseased renal parenchyma [13,14].

As a consequence, to compensate detrimental influences and to support developmental capacity a new strategy for handling of stem/progenitor cells during the process of implantation was drafted up (Figure 6b). Following this concept renal stem/progenitor cells are kept from the start of isolation, during perfusion culture, implantation and initial seeding in a medium equilibrated against atmospheric air. Applying this technique a constant fluid environment including a stabilized pH can be maintained. However, the selection of a commercially available culture medium for keeping stem/ progenitor cells under atmospheric air is problematic. Most of the culture media were designed for the use in a $\mathrm{CO}_{2}$-incubator and not for handling under laboratory air atmosphere. For that reason they contain a relative high amount of $\mathrm{NaHCO}_{3}$ provoking $\mathrm{pH}$ shifting and in turn damaging of contained cells under atmospheric air.

\section{Featuring $\mathrm{CO}_{2}$-stabilized culture media}

A technical solution to maintain $\mathrm{pH}$ might be the application of $\mathrm{CO}_{2}$-stabilized culture media. As demonstrated earlier Iscove's Modified Dulbecco's Medium buffered with HEPES, Leibovitz's $\mathrm{L}-15$ Medium and $\mathrm{CO}_{2}$ Independent Medium were shortlisted as possible candidates [24]. Consequently, in present experiments these media were tested in combination with renal stem/ progenitor cells kept in perfusion culture for 13 days under atmospheric air. Application of Iscove's Modified Dulbecco's Medium (Figure 3a), Leibovitz's L-15 Medium (Figure 3b) and $\mathrm{CO}_{2}$ Independent Medium (Figure 3c) revealed that numerous tubules are developing within the offered polyester interstitium. However, whole mount staining of specimens by SBA demonstrated that the label is not equally distributed. Most fluorescent tubules were detected in series generated in Iscove's Modified Dulbecco's Medium (Figure 3a), while less label was found in series with Leibovitz's L-15 Medium (Figure 3b). The smallest amount of labeled tubules was detected in series cultured in $\mathrm{CO}_{2}$ Independent Medium (Figure 3c).

Semithin sections further depicted that tubules contain an intact and polarized epithelium with a clearly visible lumen and a basal lamina (Figures $\mathbf{4 a}, \mathbf{5 a}$ and $\mathbf{5 d}$ ). Performing transmission electron microscopy it could be also recognized that the nucleus and cytoplasm of generated tubule cells appear normal, when stem/progenitor cells are kept in Iscove's Modified Dulbecco's Medium (Figure 4b), Leibovitz's L-15 (Figure 5b) and $\mathrm{CO}_{2}$ Independent Medium (Figure 5e). In all of the cases it was found that mitochondria are orientated more to the basal than to the apical cell side. The apical and lateral plasma membranes were found in all cases to be separated by a tight junctional complex (Figures $\mathbf{4} \mathbf{b}^{\prime}, \mathbf{5} \mathbf{b}^{\prime}$ and $\mathbf{5 e}$ '). Further the basal plasma membrane of generated tubules rests on a continuously developed basal lamina.

Most interesting are the lateral plasma membranes, since 


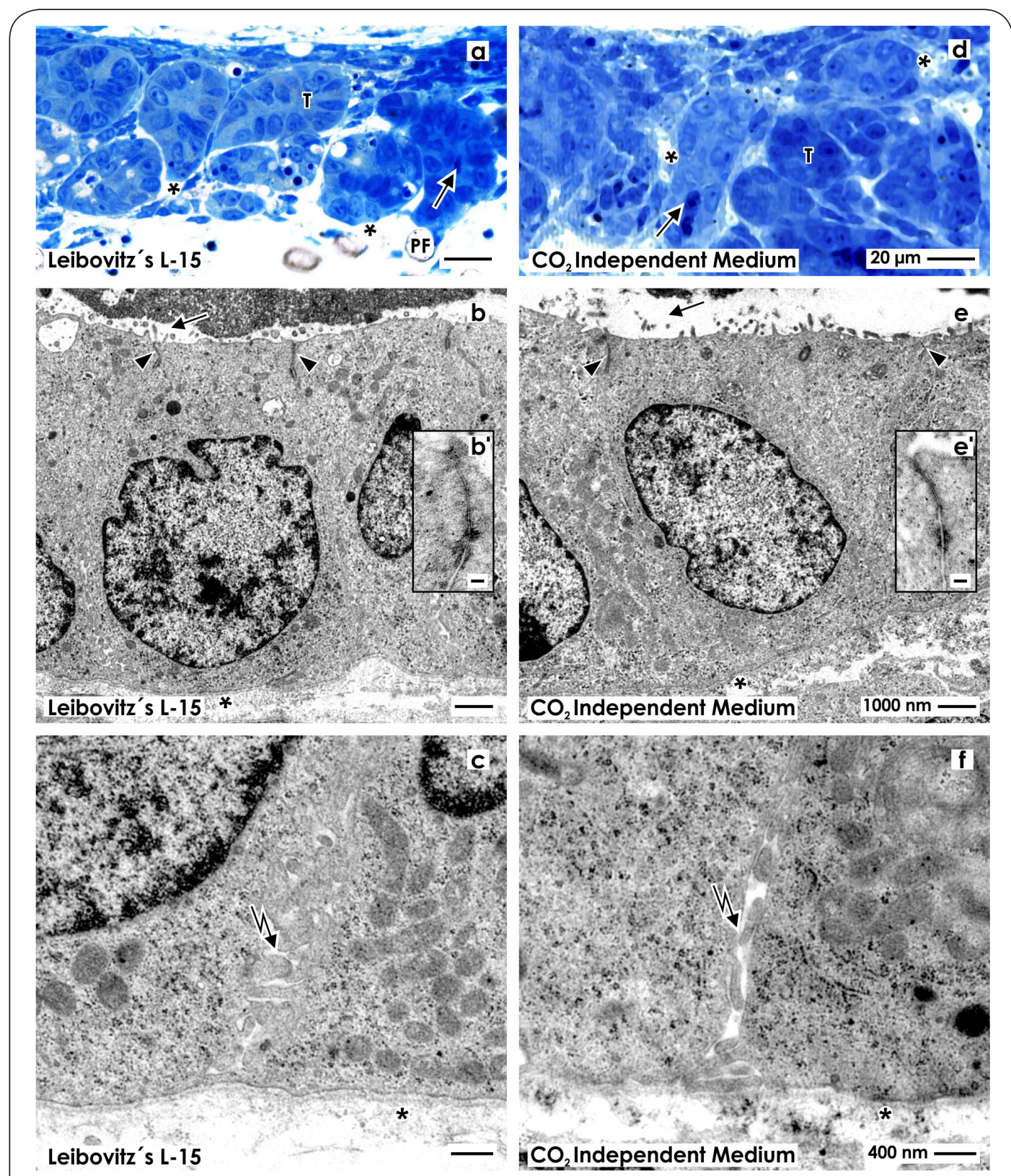

Figure 5. Light and electron microscopy of renal tubules (T) generated in Leibovitz's L-15 Medium $(\mathbf{a}, \mathbf{b}, \mathbf{c})$ and $\mathrm{CO}_{2}$ Independent Medium (d,e,f) after 13 days. (a,d) A semithin section depicts within polyester fibers (PF) numerous tubules each exhibiting a lumen and a basal lamina. (b,e) Low magnification in electron microscopy shows a polarized epithelium within generated tubules. The luminal and lateral plasma membranes are separated by a tight junctional complex (arrow head and b', e'; bar $100 \mathrm{~nm}$ ). (c,f) High magnification in electron microscopy depicts at the lateral plasma membranes intense protrusions (serrated arrow) interdigitating with each other. Lumen is indicated by an arrow, while the basal lamina is labelled by an asterisk.

they form narrow slits at the basal side speaking for an intact side to side contact of cells as it is found within the kidney. Finally, species kept in Iscove's Modified Dulbecco's Medium (Figure 4c) and Leibovitz's L-15 Medium (Figure 5c) exhibit numerous long folds respectively protrusions interdigitating with each other. In contrast, only relatively few folds were detected after culture in $\mathrm{CO}_{2}$ Independent Medium (Figure 5f). However, independently if more or less folds are detected all of the illustrated morphological features speak in favor that generation of tubules including a transporting epithelium takes place.

Finally, performed experiments demonstrated that initial 


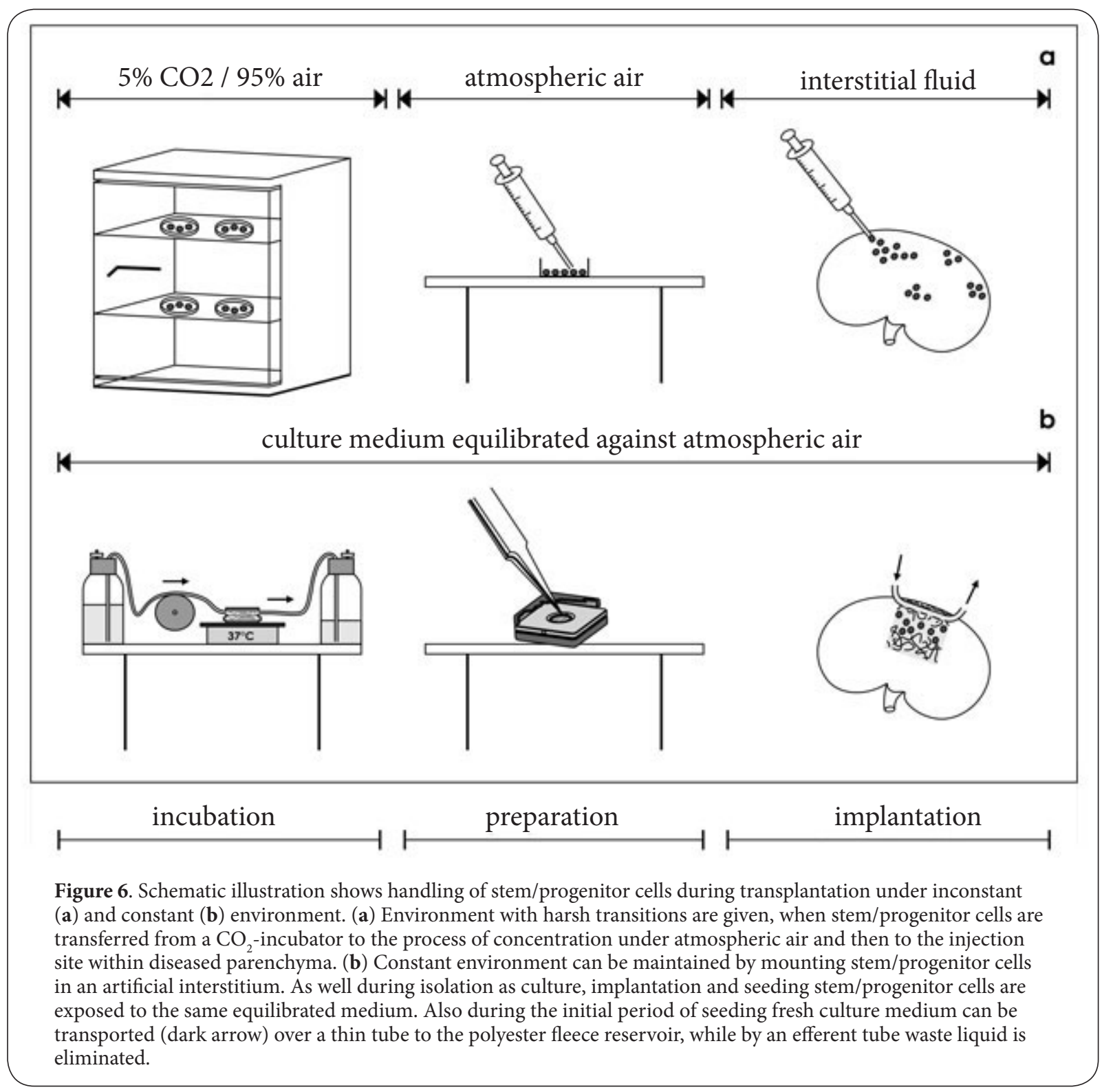

seeding of renal stem/progenitor cells and generation of renal tubules can be investigated as well with chemically defined Iscove's Modified Dulbecco's Medium (Figures 3a and 4) as Leibovitz's L-15 (Figures 3b, 5a-5c) and $\mathrm{CO}_{2}$ Independent Medium (Figures 3c, 5d-5f). According to manufacturer's information the content and chemical composition of electrolytes, amino acids, nucleotides, glucose and other compounds in applied media is not similar but very different. In so far it is surprising that all three selected media are leading to the generation of tubules exhibiting excellent morphological results. As a consequence, obtained results are a physiological mirror reflecting on the one hand a relative resistance of renal stem/progenitor cells against changeovers in fluid environment. On the other hand one has to interpret the obtained data with further care. Gross inspection for example suggests that tubules in all series look the same. However, there are gradual and barely visible differences. Experimental series with $\mathrm{CO}_{2}$ Independent Medium demonstrate in fluorescence microscopy an up to date non explainable low degree of SBA binding (Figure 3c) and in transmission electron microscopy the lowest number of protrusions on the lateral plasma membranes (Figure 5f).

Manufacturer's information further show that Iscove's Modified Dulbecco's Medium is stabilized against changes in $\mathrm{pH}$ by addition of HEPES. In contrast, Leibovitz's L-15 Medium is buffered by its complement of salts, free base and amino acids. $\mathrm{CO}_{2}$ Independent Medium is buffered by mono and dibasic sodium phosphate and $\beta$-glycerophosphate. It is obvious that applied media are not so sensitive in respect to surrounding $\mathrm{NaH}_{2} \mathrm{CO}_{3}$ and $\mathrm{CO}_{2}$ as by earlier tested Williams' $\mathrm{E}$ 
Minuth et al. Transplantation Technology 2013,

Medium, Basal Medium Eagle, McCoy's 5A Modified Medium and Medium 199 [24]. It implicates that probably not so much the chemical composition but variations in $\mathrm{pH}$ disturb proper development of tested stem/progenitor cells. However, further investigations are necessary to elucidate, if Iscove's Modified Dulbecco's Medium, Leibovitz's L-15 Medium and $\mathrm{CO}_{2}$ Independent Medium can be used without risks for implantation with stem/progenitor cells in humans.

\section{Conclusions}

To offer a constant environment during isolation, culture, surgical processing and initial seeding within diseased parenchyma, an improved concept for implantation of stem/ progenitor cells is under current work. In present experiments stabilization of fluid environment was investigated by isolating renal stem/progenitor cells in chemically defined and $\mathrm{CO}_{2}-$ stabilized culture media. The present results demonstrate that three different culture media can be short-listed for protecting stem/progenitor cells during the process of implantation. Earlier approved Iscove's Modified Dulbecco's Medium buffered by HEPES revealed formation of numerous tubules. Also application of Leibovitz's L-15 Medium and $\mathrm{CO}_{2}$ Independent Medium demonstrated unexpected promoting effects on the generation of tubules. In all series typical features of transporting tubule cells were registered. Formation of an excess of vacuoles as an indicator for cytotoxicity was not observed.

\section{Competing interests}

The authors declare that they have no competing interests. Authors' contributions

\begin{tabular}{|l|c|c|}
\hline Authors' contributions & WWM & LD \\
\hline Research concept and design & $\sqrt{ }$ & -- \\
\hline Collection and/or assembly of data & -- & $\sqrt{ }$ \\
\hline Data analysis and interpretation & $\sqrt{ }$ & $\sqrt{ }$ \\
\hline Writing the article & $\sqrt{ }$ & -- \\
\hline Critical revision of the article & $\sqrt{ }$ & $\sqrt{ }$ \\
\hline Final approval of article & $\sqrt{ }$ & $\sqrt{ }$ \\
\hline Statistical analysis & -- & $\sqrt{ }$ \\
\hline
\end{tabular}

\section{Acknowledgement}

The project was supported by the University of Regensburg.

\section{Publication history}

Editor: Dr. Elsie Maria Muller, University of Cape Town, South Africa. EIC: Dr. Stephen C Strom, Karolinska Institutet, Sweden.

Received: 12-Jul-2013 Accepted: 16-Sep-2013

Published: 28-Sep-2013

\section{References}

1. Reule $S$ and Gupta $S$. Kidney regeneration and resident stem cells. Organogenesis. 2011; 7:135-9. | Article | PubMed Abstract | PubMed Full Text

2. Harari-Steinberg O, Pleniceanu $O$ and Dekel B. Selecting the optimal cell for kidney regeneration: fetal, adult or reprogrammed stem cells.
Organogenesis. 2011; 7:123-34. | Article | PubMed Abstract | PubMed Full Text

3. Morigi $M$ and Benigni $A$. Mesenchymal stem cells and kidney repair. Nephrol Dial Transplant. 2013; 28:788-93. | Article | PubMed

4. Yokote $S$, Yamanaka $S$ and Yokoo T. De novo kidney regeneration with stem cells. J Biomed Biotechnol. 2012; 2012:453519. | Article | PubMed Abstract | PubMed Full Text

5. Das $\mathrm{R}$, Jahr H, van Osch GJ and Farrell E. The role of hypoxia in bone marrow-derived mesenchymal stem cells: considerations for regenerative medicine approaches. Tissue Eng Part B Rev. 2010; 16:15968. | Article | PubMed

6. Burst VR, Gillis M, Putsch F, Herzog R, Fischer JH, Heid P, Muller-Ehmsen J, Schenk K, Fries JW, Baldamus CA and Benzing T. Poor cell survival limits the beneficial impact of mesenchymal stem cell transplantation on acute kidney injury. Nephron Exp Nephrol. 2010; 114:e107-16. | Article I PubMed

7. Burst V, Putsch F, Kubacki T, Volker LA, Bartram MP, Muller RU, Gillis M, Kurschat CE, Grundmann F, Muller-Ehmsen J, Benzing T and Teschner $S$. Survival and distribution of injected haematopoietic stem cells in acute kidney injury. Nephrol Dial Transplant. 2013; 28:1131-9. | Article I PubMed

8. Caldas HC, Fernandes IM, Gerbi F, Souza AC, Baptista MA, Ramalho HJ, Kawasaki-Oyama RS, Goloni-Bertollo EM, Pavarino-Bertelli EC, Braile $\mathrm{DM}$ and Abbud-Filho M. Effect of whole bone marrow cell infusion in the progression of experimental chronic renal failure. Transplant Proc. 2008; 40:853-5. | Article | PubMed

9. Bussolati B and Camussi G. Stem cells in acute kidney injury. Contrib Nephrol. 2007; 156:250-8. | Article | PubMed

10. Hammerman MR. Xenotransplantation of pancreatic and kidney primordia-where do we stand? Transpl Immunol. 2009; 21:93-100. | Article | PubMed Abstract | PubMed Full Text

11. Yokoo $T$ and Kawamura T. Xenobiotic kidney organogenesis: a new avenue for renal transplantation. J Nephrol. 2009; 22:312-7. | PubMed

12. Kim SS, Park HJ, Han J, Gwak SJ, Park MH, Song KW, Rhee YH, Min Chung $\mathrm{H}$ and Kim BS. Improvement of kidney failure with fetal kidney precursor cell transplantation. Transplantation. 2007; 83:1249-58. | Article I PubMed

13. Ricardo SD, van Goor $\mathrm{H}$ and Eddy AA. Macrophage diversity in renal injury and repair. J Clin Invest. 2008; 118:3522-30. | Article | PubMed Abstract | PubMed Full Text

14. Zoja C, Garcia PB and Remuzzi G. The role of chemokines in progressive renal disease. Front Biosci (Landmark Ed). 2009; 14:1815-22. | Article | PubMed

15. Liu Y. Cellular and molecular mechanisms of renal fibrosis. Nat Rev Nephrol. 2011; 7:684-96. | Article | PubMed

16. Lee DW, Faubel $S$ and Edelstein CL. Cytokines in acute kidney injury (AKI). Clin Nephrol. 2011; 76:165-73. | Article | PubMed

17. Chung AC and Lan HY. Chemokines in renal injury. J Am Soc Nephrol. 2011; 22:802-9. | Article | PubMed

18. Palm F and Nordquist L. Renal tubulointerstitial hypoxia: cause and consequence of kidney dysfunction. Clin Exp Pharmacol Physiol. 2011; 38:474-80. | Article | PubMed Abstract | PubMed Full Text

19. Chiang CK, Tanaka T and Nangaku M. Dysregulated oxygen metabolism of the kidney by uremic toxins: review. J Ren Nutr. 2012; 22:77-80. | Article I PubMed

20. Bonventre JV and Yang L. Cellular pathophysiology of ischemic acute kidney injury. J Clin Invest. 2011; 121:4210-21. | Article | PubMed Abstract I PubMed Full Text

21. Miyamoto T, Carrero JJ and Stenvinkel P. Inflammation as a risk factor and target for therapy in chronic kidney disease. Curr Opin Nephrol Hypertens. 2011; 20:662-8. | Article | PubMed

22. Minuth WW, Denk $L$ and Glashauser A. Cell and drug delivery therapeutics for controlled renal parenchyma regeneration. Adv Drug Deliv Rev. 2010; 62:841-54. | Article | PubMed

23. Minuth WW and Denk L. Interstitial interfaces show marked differences in regenerating tubules, matured tubules, and the renal stem/ 
Minuth et al. Transplantation Technology 2013,

http://www.hoajonline.com/journals/pdf/2053-6623-1-2.pdf

progenitor cell niche. J Biomed Mater Res A. 2012; 100:1115-25. | Article

I PubMed

24. Minuth WW, Denk $L$ and Gruber M. Search for chemically defined culture medium to assist initial regeneration of diseased renal parenchyma after stem/progenitor cell implantation. Int J Stem Cell Res Transplant. 2013; 1:202. I Pdf

25. Roessger A, Denk L and Minuth WW. Potential of stem/progenitor cell cultures within polyester fleeces to regenerate renal tubules. Biomaterials. 2009; 30:3723-32. I Article | PubMed

26. Minuth WW, Denk L, Meese C, Rachel R and Roessger A. Ultrastructural insights in the interface between generated renal tubules and a polyester interstitium. Langmuir. 2009; 25:4621-7. | Article | PubMed

27. Minuth WW, Denk L, Miess C and Glashauser A. Peculiarities of the extracellular matrix in the interstitium of the renal stem/progenitor cell niche. Histochem Cell Biol. 2011; 136:321-34. | Article | PubMed

\section{Citation:}

Minuth WW and Denk L. Initial steps to stabilize the microenvironment for implantation of stem/progenitor cells in diseased renal parenchyma. Transplant Technol. 2013; $1: 2$.

http://dx.doi.org/10.7243/2053-6623-1-2 